\title{
Psoralen, a natural phytoestrogen, improves diaphyseal fracture healing in ovariectomized mice: A preliminary study
}

\author{
KUI HUANG $^{1 *}$, YA-QIONG SUN ${ }^{2 *}$, XIAO-FENG CHEN ${ }^{3}$, FENG TIAN $^{1}$, \\ FAN CHENG $^{1}$, QIAN-LONG GONG ${ }^{1}$ and KE-BIN LIU ${ }^{1}$
}

\author{
Departments of ${ }^{1}$ Orthopedics and ${ }^{2}$ Imaging, The First Hospital of Yangtze University, Jingzhou, Hubei 434000; \\ ${ }^{3}$ Department of Orthopedics, Panyu Hospital of Chinese Medicine, Guangzhou, Guangdong 511400, P.R. China
}

Received September 16, 2019; Accepted September 16, 2020

DOI: $10.3892 /$ etm.2021.9799

\begin{abstract}
Psoralen is an effective active component extracted from Psoraleacorylifolia, which can promote bone formation in osteoporotic animals. However, to the best of our knowledge, its effect on fracture healing has not yet been examined. In the present study, open femur fractures were created in ovariectomy (OVX)-induced osteoporotic mice. OVX mice were treated with psoralen (psoralen+OVX group) or physiological saline (OVX group) by oral gavage. Radiographic and histological results demonstrated progressed callus consolidation in the psoralen+OVX group compared with the OVX group after 10 and 21 days of treatment. Qualitative histological analysis showed that the number of osteoclasts was significantly reduced in the psoralen+OVX group after treatment. Moreover, reverse transcription-quantitative PCR analysis of callus samples showed increased expression of bone morphogenetic protein-2 (BMP-2) and osteoprotegerin (OPG), and decreased expression of receptor activator of nuclear factor $-\kappa B$ ligand (RANKL) at 10 and 21 days post injury in the psoralen+OVX group compared with the OVX group. Furthermore, western blot analysis showed that psoralen significantly increased the expression of estrogen receptor (ER)- $\alpha$, but had no effect on ER- $\beta$ expression; these results were further confirmed by immunohistochemistry. To conclude, these results indicated that psoralen may promote callus formation and inhibit
\end{abstract}

Correspondence to: Dr Ke-Bin Liu, Department of Orthopedics, The First Hospital of Yangtze University, 8 Aviation Road, Jingzhou, Hubei 434000, P.R. China

E-mail: liukb@sina.com

*Contributed equally

Abbreviations: OVX, ovariectomy; BMP-2, bone morphogenetic protein-2; OPG, osteoprotegerin; RANKL, receptor activator of nuclear factor- $\mathrm{kB}$ ligand; ER, estrogen receptor; TRAP, tartrate-resistant acid phosphatase; $\mathrm{BV}$, bone volume; TV, tissue volume; TMD, tissue mineral density; H\&E, hematoxylin and eosin

Key words: osteoporosis, fracture healing, ovariectomy, mice, phytoestrogens osteoclast genesis by increasing BMP-2 and ER- $\alpha$ levels, and OPG/RANKL ratio. Consequently, psoralen could be a possible treatment for osteoporotic fracture-related complications.

\section{Introduction}

Osteoporotic fractures are the most severe complications related to osteoporosis, which is characterized by skeletal fragility and microarchitectural deterioration of bone tissue (1). Previous studies have shown that elderly female patients are at a higher risk of delayed union and nonunion fractures (2); thus, alternative techniques and treatments for osteoporotic fractures have gained increasing research interest over recent years.

Antiresorptive pharmacological agents, such as bisphosphonates and estrogen, can improve bone health and reduce the risk of fractures (3). Nevertheless, their effect on fracture healing remains unclear. For example, bisphosphonates cannot stimulate new bone formation, and long-term use of bisphosphonates can delay fracture healing and lead to a prolonged presence of callus and delayed callus remodeling $(4,5)$. Therefore, the delayed administration of bolus-dose bisphosphonates may be optimal for the treatment of fragility fractures $(6,7)$. Due to an increased risk of breast carcinoma, endometrial cancer and cardiovascular disease, estrogen is no longer recommended as first-line therapy for osteoporosis according to new guidelines $(8,9)$. Additionally, due to the adverse effects related to synthetic drugs, there is an increased demand for alternative or natural medicines for the treatment of osteoporosis and associated complications. Previous studies have shown that phytoestrogens, which can be found in certain traditional Chinese medicines, have anti-osteoporotic potential (10-12).

Psoralen is an active component extracted from Psoraleacorylifolia that belongs to the phytoestrogen group of the furanocoumarin class of compounds (13). Previous in vitro studies have revealed that psoralen may induce the proliferation of osteoblasts, increase the expression of osteoprotegerin (OPG) and decrease the expression levels of receptor activator of nuclear factor- $\kappa \mathrm{B}$ ligand (RANKL) to suppress the differentiation and maturation of osteoclasts $(14,15)$. In sex hormone deficiency-induced osteoporosis animal models, the administration of psoralen 
at different doses (10 and $20 \mathrm{mg} / \mathrm{kg}$ ) significantly increased the values of alkaline phosphatase/tartrate-resistant acid phosphatase (TRACP) and bone volume (BV)/tissue volume (TV) in intact bone, exhibiting anti-osteoporotic effects in female and male mice after 8 weeks (16). Furthermore, in a previous study by the authors of the current study, it was found that the administration of $20 \mathrm{mg} / \mathrm{kg}$ psoralen for 6 weeks could increase the levels of serum alkaline phosphatase and improve bone biomechanics in ovariectomized (OVX) mice (17). Due to these properties, psoralen could be considered as an alternative option for the management of osteoporosis; nevertheless, to the best of our knowledge, its effect on fracture healing has not yet been examined.

In the present study, a standardized femoral osteotomy model and bone fixation with an intramedullary pin was used in the OVX mouse model to mimic the condition in post-menopausal women. The effects of psoralen on the osteoporotic fracture healing process and the underlying mechanisms were examined.

\section{Materials and methods}

Establishment of an osteoporosis model in mice. A total of 7-10-week-old, $20 \mathrm{~g}$, female C57BL/6 mice were obtained from the Hubei Research Center of Laboratory Animals. All animals were housed in an environment with a temperature of $22 \pm 1^{\circ} \mathrm{C}$, a relative humidity of $50 \pm 1 \%$ and a light/dark cycle of $12 / 12 \mathrm{~h}$. Animals were allowed free access to water and food pellets. In addition, all animal studies, including the euthanasia procedure, were approved and performed in compliance with the regulations and guidelines of the Medical Ethical Committee of the First Affiliated Hospital of Yangtze University (approval no. 2017-060; Jingzhou, China) and conducted according to The Association for Assessment and Accreditation of Laboratory Animal Care International and the Institutional Animal Care and Use Committee guidelines (18).

When the mice reached 12 weeks of age, 10 mice were used initially to check whether the OVX model could be successfully established and subsequently, 60 mice were used for further experiments. A total of 10 mice were randomly divided into two groups ( $n=5 /$ per group): Sham-operated group and OVX group. Under anesthesia, the sham-operated group mice were subjected to a bilateral laparotomy, without affecting the ovaries. OVX group was subject to bilateral OVX using the dorsal approach. After surgery, mice were left untreated for 6 weeks, following which all the mice in each group were sacrificed. The successful establishment of the OVX model was confirmed via atrophy of gonads and femoral metaphysis determined via histological evaluation.

Experimental design. Once the successful establishment of the OVX model was confirmed, 60 OVX mice were divided into two subgroups: i) OVX group, treated with physiological saline solution; and ii) psoralen+OVX group treated with $20 \mathrm{mg} / \mathrm{kg}$ psoralen ( $\geq 98 \%$ purity; Yongjian Pharmaceutical Co., Ltd.). These drug doses (20 mg/kg psoralen) have been shown to promote bone metabolism and biomechanics in ovariectomized mice (14). Under anesthesia, a standardized fracture was created on the mid diaphysis of the left femur in the OVX and psoralen+OVX groups, and stabilized with an intramedullary pin, as described below. After osteotomy, saline and psoralen treatments were given via an oral gavage route for a total of 21 days.

Surgical protocol. Mice were anesthetized by intraperitoneal injection of ketamine hydrochloride (100 mg/ $\mathrm{kg}$ body weight) and xylazine $(20 \mathrm{mg} / \mathrm{kg}$ body weight). After successful anesthesia and sterilization, the skin and thigh muscle were incised to expose the midshaft of the femur. Transverse-shaped osteotomy was performed on the middle femoral shaft with a $0.3-\mathrm{mm}$ diameter micro-high-speed milling cutter. At the same time, saline solutions were used for local washing and cooling. Then, the fracture was reduced, following which a retrograde intramedullary pin fixation $(0.55 \mathrm{~mm}$ in diameter $)$ was performed. The muscular layer and skin were sutured. All of the animals received an intramuscular antibiotic $(10 \mathrm{mg} / \mathrm{kg}$ penicillin) and analgesic ( $1 \mathrm{mg} / \mathrm{kg}$ meloxicam) injection for three days after the operation.

Psoralen. Psoralen powder was ground and suspended in physiological saline to achieve a final concentration of $2 \mathrm{mg} / \mathrm{ml}$. The psoralen+OVX group was treated with psoralen at a dose of $20 \mathrm{mg} / \mathrm{kg} / \mathrm{day}$ from the first post operative day.

Specimen collection and preparation. Mice were sacrificed at 10 days ( $n=15 /$ per group) or 21 days $(n=15 /$ per group) post-surgery to assess the endochondral ossification phase and the bone remodeling stage of fracture healing. The osteotomized femurs were evaluated using X-ray, micro-CT, histology and immunohistochemistry. Fracture callus tissues were harvested for reverse transcription-quantitative (RT-q) PCR and western blot analysis at 10 and 21 days.

$X$-ray and micro-CT analysis of fracture healing. Radiographs were taken 21 days after fracture to evaluate the fracture healing, using an X-ray mammography system (Siemens AG) with an exposure of $39 \mathrm{kV}$ for $32 \mathrm{sec}$. On day 21, micro-CT was performed using a Scanco Micro-CT 60 system (Scanco Medical AG) and a previously described method (19). Briefly, the range covered $10 \mathrm{~mm}$ of the callus; the fracture in the center was scanned and images were reconstructed to nominal $12-\mathrm{mm}$ voxel size. Only the newly formed callus was used to define the tissue perimeters as the volume of interest. Two fixed, global thresholds with the lower limit corresponding to a mineral density of $421 \mathrm{mg}$ hydroxyapatite $/ \mathrm{cm}^{3}$ and the upper limit corresponding to $3,000 \mathrm{mg}$ hydroxyapatite $/ \mathrm{cm}^{3}$ were used to distinguish mineralized from non-mineralized tissues. Quantitative parameters used for evaluation included TV, BV, $\mathrm{BV} / \mathrm{TV}$ and tissue mineral density (TMD).

Histology and immunohistochemical examination. The fractured femurs were harvested. Soft tissue was removed without disturbing the callus, and the fractured femurs were fixed in $10 \%$ buffered formalin for $48 \mathrm{~h}$ in $4^{\circ} \mathrm{C}$. The formalin-fixed samples were decalcified in $10 \%$ EDTA and then embedded in paraffin. Sections of $5 \mu \mathrm{m}$ were cut in the sagittal plane, and analyzed using the following approach. Samples were stained with hematoxylin and eosin (H\&E) for basic morphology or with tartrate-resistant acid phosphatase 
(TRAP) to analyze the osteoclast cells. In the same mice, a total of six slides were analyzed. Three specimens were used for HE staining and TRAP staining, respectively. Osteoclasts were identified as TRACP+cells with $\geq$ three nuclei. Finally, three fields of view (magnification, x200) were randomly selected for each slice. The number of stained cells in each field was calculated using Image-Pro Plus 6.0 analysis software (Media Cybernetics, Inc.). Immunohistochemical staining included a standard immunohistochemical procedure for bone morphogenetic protein-2 (BMP-2), estrogen receptor (ER)- $\alpha$ and ER- $\beta$ protein expression that was performed on the paraffin sections obtained on day 21 . In brief, Paraffin sections were dewaxed by successively placing the slices into xylene I, II and III (each for $15 \mathrm{~min}$ ), and then water ethanol I, absolute ethanol II, 85\% alcohol and $75 \%$ alcohol (each for $5 \mathrm{~min}$ ). Samples were then washed with distilled water. Tissue sections were treated with citric acid antigen repair buffer $(\mathrm{pH}$ 6.0) in a microwave oven for antigen retrieval. Samples were subjected to moderate heating in the microwave for $8 \mathrm{mins}$ to boil, left to rest for $8 \mathrm{~min}$ and then turned to medium low heating for $7 \mathrm{~min}$. This process should prevent the buffer excessive evaporation, do not dry tablets. After natural cooling, the slides were placed in PBS ( $\mathrm{pH}$ 7.4) and shaken on the decolorizing shaker. The samples were washed in PBS ( $\mathrm{pH} 7.4)$ in triplicate, with each wash lasting for $5 \mathrm{~min}$. Endogenous peroxidase was quenched using 3\% hydrogen peroxide for $10 \mathrm{~min}$, and nonspecific binding of epitopes was blocked using 3\% BSA for $30 \mathrm{~min}$ at room temperature. Slides were incubated at $4^{\circ} \mathrm{C}$ overnight with the primary antibodies for BMP-2 (1:500; cat. no. GB11252), ER- $\alpha$ (1:200; cat. no. GB13205) and ER- $\beta$ (1:500; cat. no. GB13003), all purchased from Wuhan Servicebio Technology Co., Ltd. Tissues were incubated at room temperature for $50 \mathrm{~min}$ with goat anti-mouse IgG secondary antibodies (cat. no. G1214; Wuhan Servicebio Technology Co., Ltd.; 1:200;) and Morphometric analysis was performed by measuring the average relative optical staining areas using Image-Pro Plus 6.0 analysis software.

All stained sections were analyzed under a light microscope (Leica RM2156; Leica Microsystems GmbH; magnification, $\mathrm{x} 200)$. The region of interest was selected as the center of the fracture, encompassing an entire cross-sectional area of a callus.

Callus mRNA quantification by RT-qPCR. Total RNA was extracted from the fracture callus and $1 \mathrm{~mm}$ of normal bone margin from each femur, and purified using RNA extracting solution (cat. no. G3013; Wuhan Servicebio Technology Co., Ltd.). The total RNA concentration was determined using a NanoDrop $^{\text {TM }} 2000$ (NanoDrop Technologies; Thermo Fisher Scientific, Inc.), then stored at $-80^{\circ} \mathrm{C}$ until further processing. Reverse transcription was performed with a commercial RevertAid First Strand cDNA Synthesis kit (cat. no. K1622; Thermo Fisher Scientific, Inc.) according to the manufacturer's protocol. Relative quantification of mRNA was performed using FastStart Universal SYBR-Green Master (Rox) (cat. no. 04913914001; Roche Diagnostics GmbH) and ABI real-time PCR System (StepOnePlus ${ }^{\mathrm{TM}}$; Applied Biosystems; Thermo Fisher Scientific, Inc.). RT-qPCR assays for BMP-2, RANKL and OPG were performed using the primer sequences listed in Table I. All gene expression levels were normalized to $\beta$-actin. The thermocycling conditions for qPCR were as follows: Initial denaturation at $95^{\circ} \mathrm{C}$ for $2 \mathrm{~min}$; followed by 40 cycles of denaturation at $95^{\circ} \mathrm{C}$ for $10 \mathrm{sec}$, annealing and elongation at $60^{\circ} \mathrm{C}$ for $30 \mathrm{sec}$; with a final extension step at $72^{\circ} \mathrm{C}$ for $30 \mathrm{sec}$. For qPCR, cDNA was used for amplification of the target genes in triplicate. Relative levels of BMP-2, RANKL and OPG were measured using $\beta$-actin as a reference gene (20). Expression levels were determined using the $2^{-\Delta \Delta C}$ q formula (21).

Western blotting. The callus tissue was lysed in RIPA buffer (cat. no. G2002; Wuhan Servicebio Technology Co., Ltd.) and protease inhibitor cocktail (cat. no. G2006; Wuhan Servicebio Technology Co., Ltd.). Protein concentrations were determined using the BCA protein quantitative detection kit according to the manufacturer's instructions (cat. no. G2026; Wuhan Servicebio Technology Co., Ltd.). Equivalent amounts of protein $(25 \mu \mathrm{g})$ were size-fractionated using $10 \%$ SDS-PAGE (cat. no. G2003; Wuhan Servicebio Technology Co., Ltd.) and electrotransferred onto Protran ${ }^{\circledR}$ nitrocellulose membranes (cat. no. IPVH00010; EMD Millipore). The membranes were then blocked with 5\% non-fat milk in Tris-buffered saline for $10 \mathrm{~h}$ at room temperature. Consequently, the membranes were incubated at $4^{\circ} \mathrm{C}$ overnight with anti-mouse ER- $\alpha$ (cat. no. MS-354-P0; Thermo Fisher Scientific, Inc.; 1:1,000), rabbit anti-mouse ER- $\beta$ (cat. no. 14007-1-AP; ProteinTech Group, Inc.; 1:1,000) and anti-mouse $\beta$-actin (cat. no. GB12001; Wuhan Servicebio Technology Co., Ltd.; 1:3,000) antibodies, and subsequently with certain horseradish peroxidase-conjugated secondary antibodies, including ER- $\alpha$ (cat. no. GB11205; Wuhan Servicebio Technology Co., Ltd. 1:3,000), $\beta$-actin (cat. no. GB12001, Wuhan Servicebio Technology Co., Ltd. 1:3,000) and ER- $\beta$ (cat. no. 14007-1-AP; Wuhan Sanying Biotechnology. $1: 3,000$ ) at room temperature for $30 \mathrm{~min}$. Proteins were visualized using the enhanced chemiluminescence detection system solution (EMD Millipore). The intensity of the specific bands was semi-quantified by densitometry using Alpha Innotech software (Version 4.0.0; FluorChem HD2; PoteinSimple).

Statistical analysis. Values are presented as the mean \pm SD of three independent repeats. All statistical comparisons were performed using SPSS 17.0 software for Windows (SPSS, Inc.). For normally distributed data, the statistical analysis was performed with a Student's t-test. Data that were not normally distributed were analyzed using the Mann-Whitney U test. $\mathrm{P}<0.05$ was considered to indicate a statistically significant difference.

\section{Results}

Confirmation of osteoporotic model. The successful establishment of the osteoporotic model was evaluated by uterine atrophy and femoral metaphysis histology. After six weeks, bilateral OVX led to marked gonadal hypertrophy in OVX mice compared with the sham mice (Fig. 1A). In addition, $\mathrm{H} \& \mathrm{E}$ staining images of femoral metaphysis in OVX mice showed that the bone trabeculae had notably higher separation and was sparsely arranged compared to the sham group 
Table I. Oligonucleotide primer sequences for reverse transcription-quantitative PCR analysis of gene expression.

\begin{tabular}{lcc}
\hline Gene & NCBI accession number & Sequence $\left(5^{\prime} \rightarrow 3^{\prime}\right)$ \\
\hline$\beta$-actin & NM_007393.3 & F: GTGACGTTGACATCCGTAAAGA \\
BMP-2 & NM_007553.3 & R: GTAACAGTCCGCCTAGAAGCAC \\
& & F: CGAATTTGAGTTGAGGCTGCTC \\
RANKL & NM_011613.3 & R: GCCGTTTTCCCACTCATCTCT \\
OPG & NM_008764.3 & F: CCATCGGGTTCCCATAAAGTCA \\
& & R: CAGTTTTCGTGCTCCCTCCTT \\
& & F: CCAGATGGGTTCTTCTCAGGTG \\
& R:GTCCACCAAAACACTCAGCCAA
\end{tabular}

NCBI, National Center for Biotechnology Information; F, forward; R, reverse; BMP-2, bone morphogenetic protein-2; RANKL, receptor activator of nuclear factor- $\mathrm{\kappa B}$ ligand; OPG, osteoprotegerin.

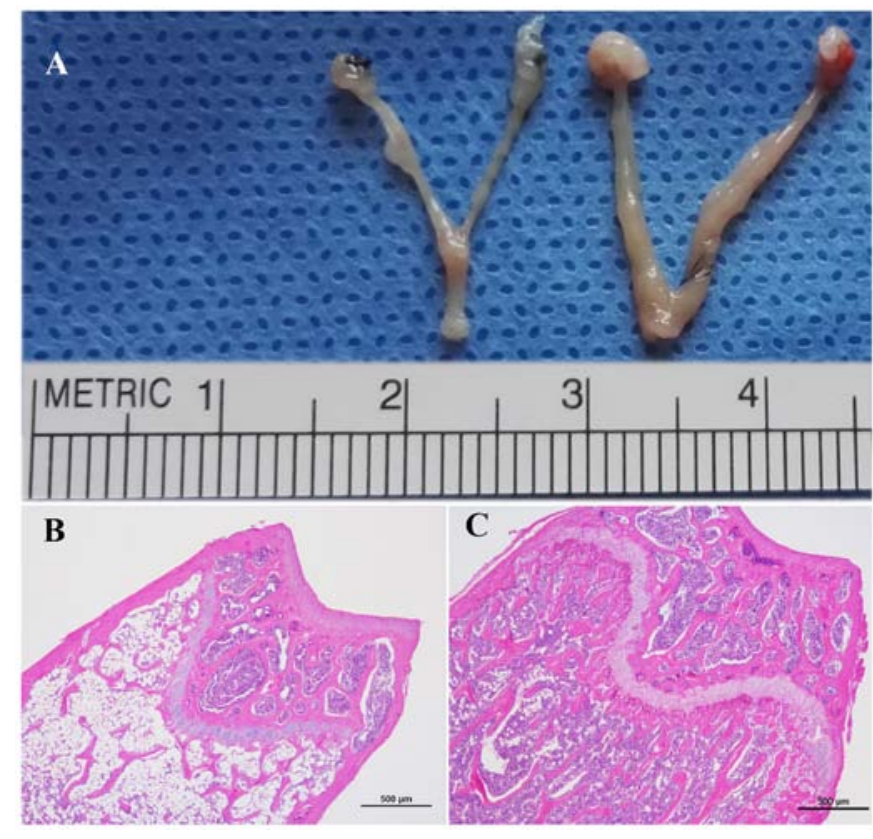

Figure 1. Successful establishment of the osteoporosis model. (A) Effectiveness of bilateral OVX was determined by observing gonadal hypertrophy in OVX mice (left) compared with the sham mice (right). Effect of bilateral OVX observed in hematoxylin and eosin staining images of femoral metaphysis in (B) OVX mice and (C) sham mice. Magnification, $\mathrm{x} 4$. OVX, ovariectomy.

(Fig. 1B and C), which further confirmed that the osteoporotic model was successfully established.

Radiographic healing and callus histology. Twenty-one days after fracture, the callus of the psoralen+OVX group mice appeared radiopaque, compared with OVX group mice (Fig. 2A). Furthermore, representative 3D views of the callus structure demonstrated that an intact periosteal callus appeared to bridge the fracture in psoralen+OVX group mice, compared with the OVX group in which bony callus development and cortical bridging were notably disrupted in the mice (Fig. 2B and C). Data for quantitative parameters are shown in Fig. 2D-G. The TV was similar between the psoralen+OVX and OVX groups, which suggested that psoralen treatment did not affect fracture callus size. However, BV and BV/TV were significantly increased in the calluses of the psoralen+OVX group compared with the OVX group $(\mathrm{P}<0.05)$.

Compared with the OVX group, histological data showed more rapid endochondral ossification and bone formation in the psoralen+OVX group. On day 10, the psoralen+OVX group showed increased mineralized callus tissue and new bone formation in the callus (Fig. 3A and B). There was also an apparent difference in bone and cartilage composition between psoralen+OVX and OVX groups on day 21. The psoralen+OVX group showed an increased maturation and mineralization of callus at the fracture gap while abundant cartilage persisted in the OVX group (Fig. 3C and D).

Effect of psoralen treatment on BMP-2 expression. Results of RT-qPCR analyses for gene expression showed that there was increased BMP-2 expression at days 10 and 21 in the psoralen+OVX group compared with the OVX group (Fig. 4D and E). These data were further confirmed by immunohistochemistry findings at the protein level; after 21 days, immunohistochemical staining showed higher signal intensities for BMP-2 in the psoralen+OVX group compared with the OVX group (Fig. 4A-C).

Effect of psoralen treatment on osteoclasts. The callus stained with TRAP at 21 days showed that TRAP-positive multinucleated giant cells were easily observed in the OVX group along with the newly formed woven bone tissue (Fig. 5A and B). By contrast, the number of osteoclasts was significantly reduced in the psoralen+OVX group (Fig. 5C).

The mRNA expression levels of OPG and RANKL in fracture calluses were determined to evaluate the potential mechanisms of osteoclast activity. On days 10 and 21, increased OPG gene expression levels and decreased expression levels of RANKL were observed in the psoralen+OVX group compared with the OVX group (Fig. 4D and E).

Effect of psoralen treatment on ER. Western blot analysis of ER- $\alpha$ and ER- $\beta$ expression levels in the callus showed that psoralen was able to significantly increase ER- $\alpha$, but had no effect on ER- $\beta$ expression (Fig. 6G-J). These results were further confirmed by immunohistochemistry. ER- $\alpha$ was 
A

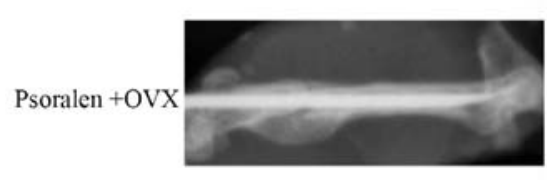

ovx

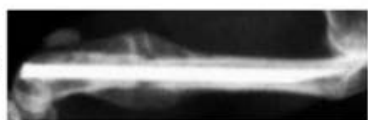

$\mathrm{D}$
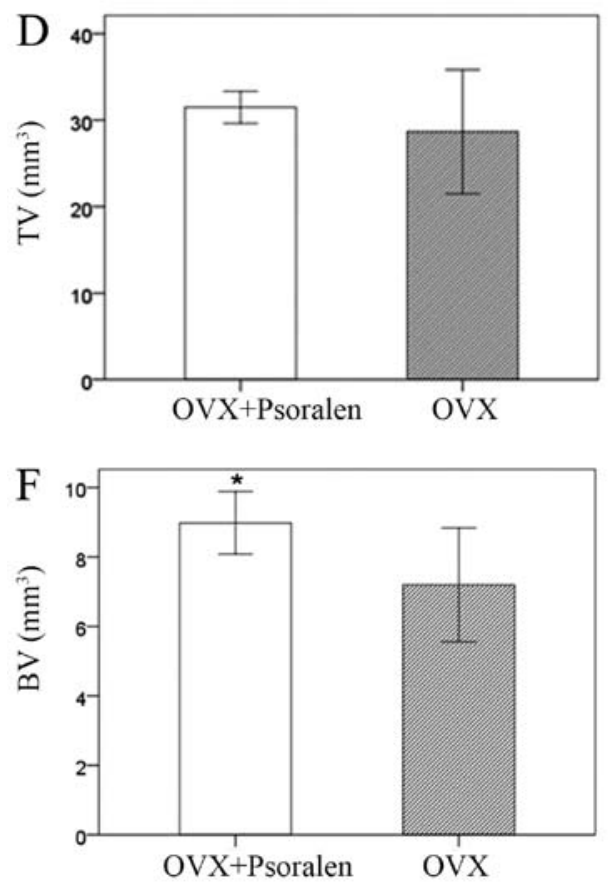

B
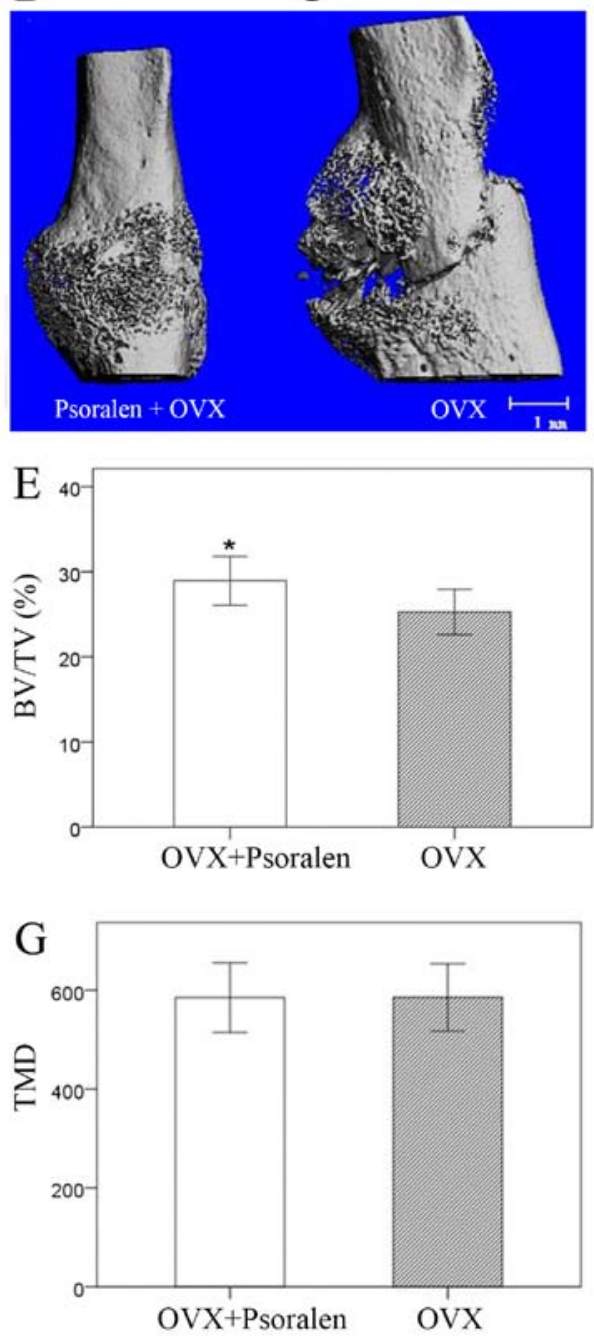

Figure 2. X-ray and micro-CT demonstrated fracture healing. (A) Representative X-ray images. 3D images generated from micro-CT analysis of bone healing in (B) psoralen+OVX group and (C) OVX group 21 days after fracture. Mean levels of (D) TV, (E) BV/TV, (F) BV and (G) TMD were obtained. Data are presented as the mean $\pm \mathrm{SD}$. " $\mathrm{P}<0.05$ vs. OVX group. OVX, ovariectomy; TV, tissue volume; BV, bone volume; TMD, tissue mineral density.

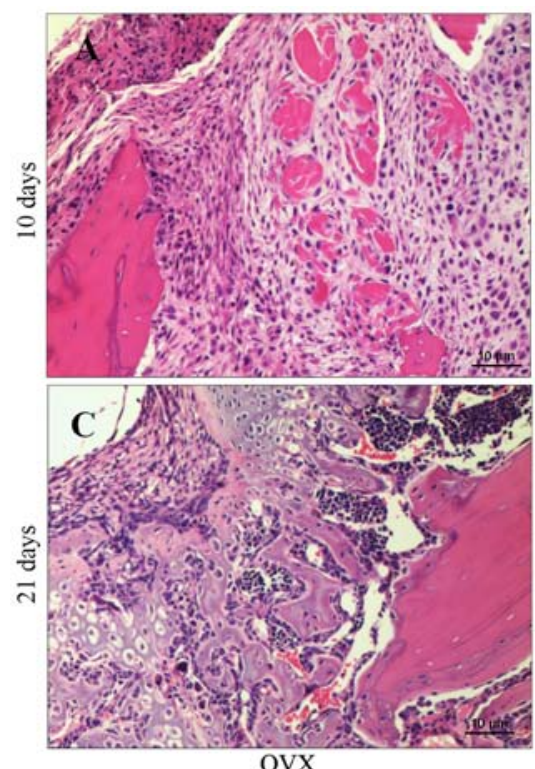

OVX
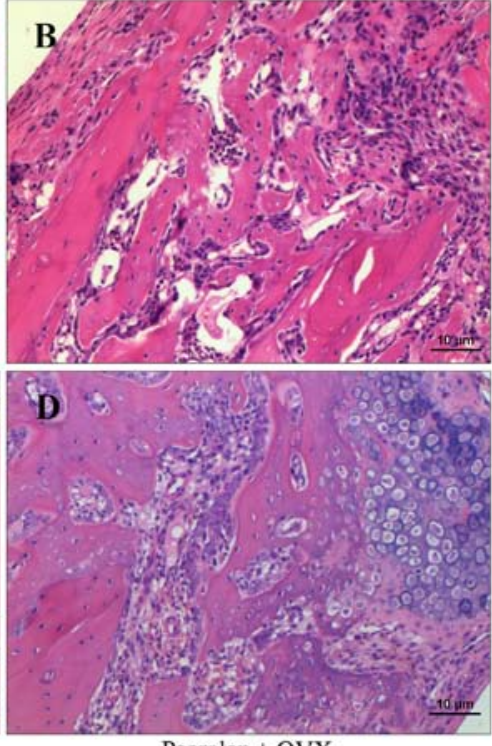

Psoralen + OVX

Figure 3. HE staining demonstrated fracture healing. Representative histological images 10 days after fracture for the (A) OVX group and (B) psoralen+OVX. Representative histological images 21 days after fracture for the (C) OVX group and (D) psoralen+OVX group. Sections were stained using the hematoxylin and eosin. Magnification, x10. OVX, ovariectomy. 

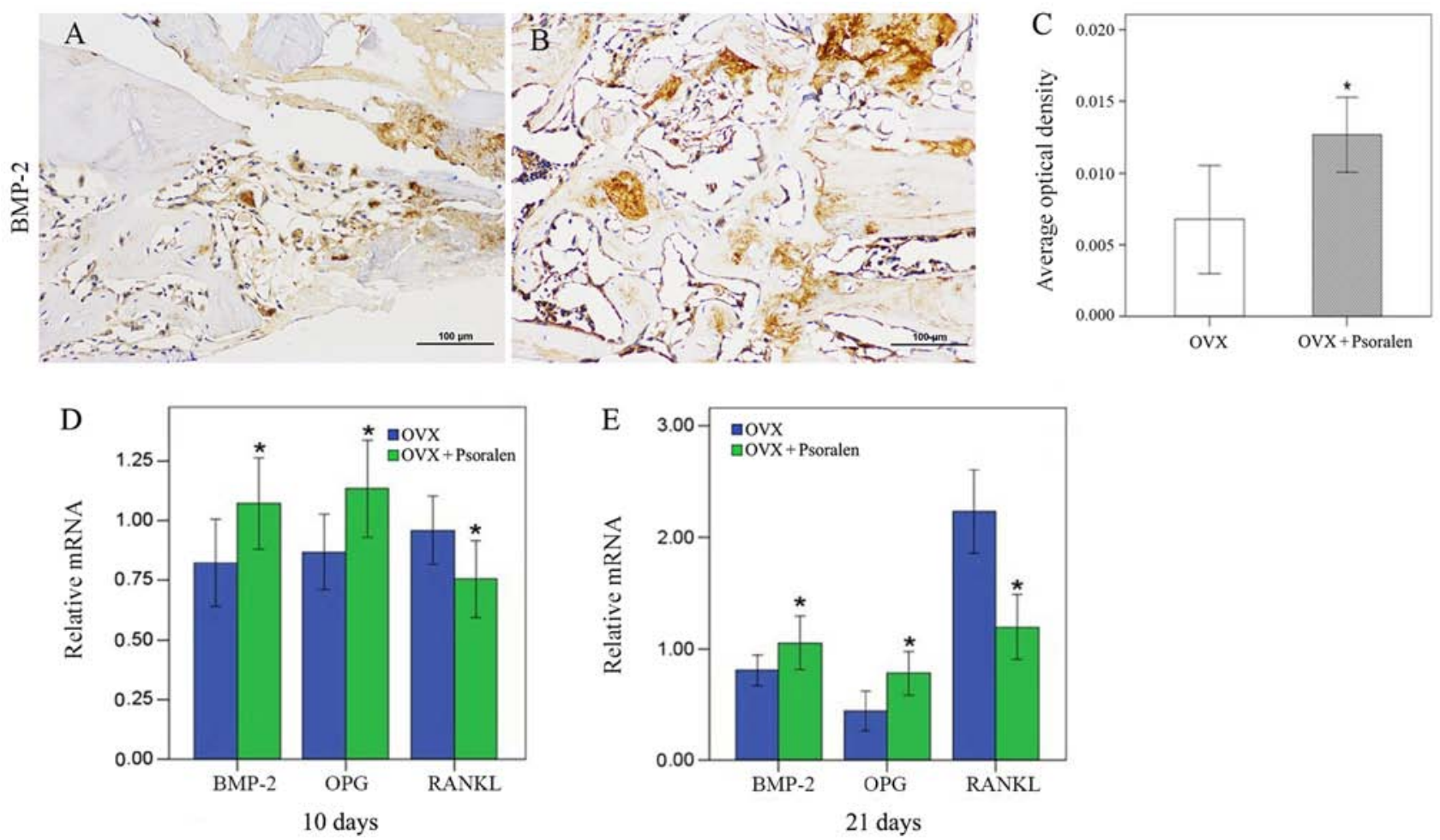

Figure 4. Expression of genes and proteins associated with osteogenesis in callus. Immunohistochemical staining for BMP-2 in (A) OVX group and (B) psoralen+OVX group. (C) Average optical density was used for the quantification of BMP-2 expression. Magnification, x20. BMP-2, OPG and RANKL mRNA expression was determined by reverse transcription-quantitative PCR in callus samples at (D) 10 and (E) 21 days post fracture. Data are presented as the mean \pm SD. " $\mathrm{P}<0.05$ vs. OVX group. OVX, ovariectomy; BMP-2, bone morphogenetic protein-2; OPG, osteoprotegerin; RANKL, receptor activator of nuclear factor- $\mathrm{\kappa} B$ ligand.
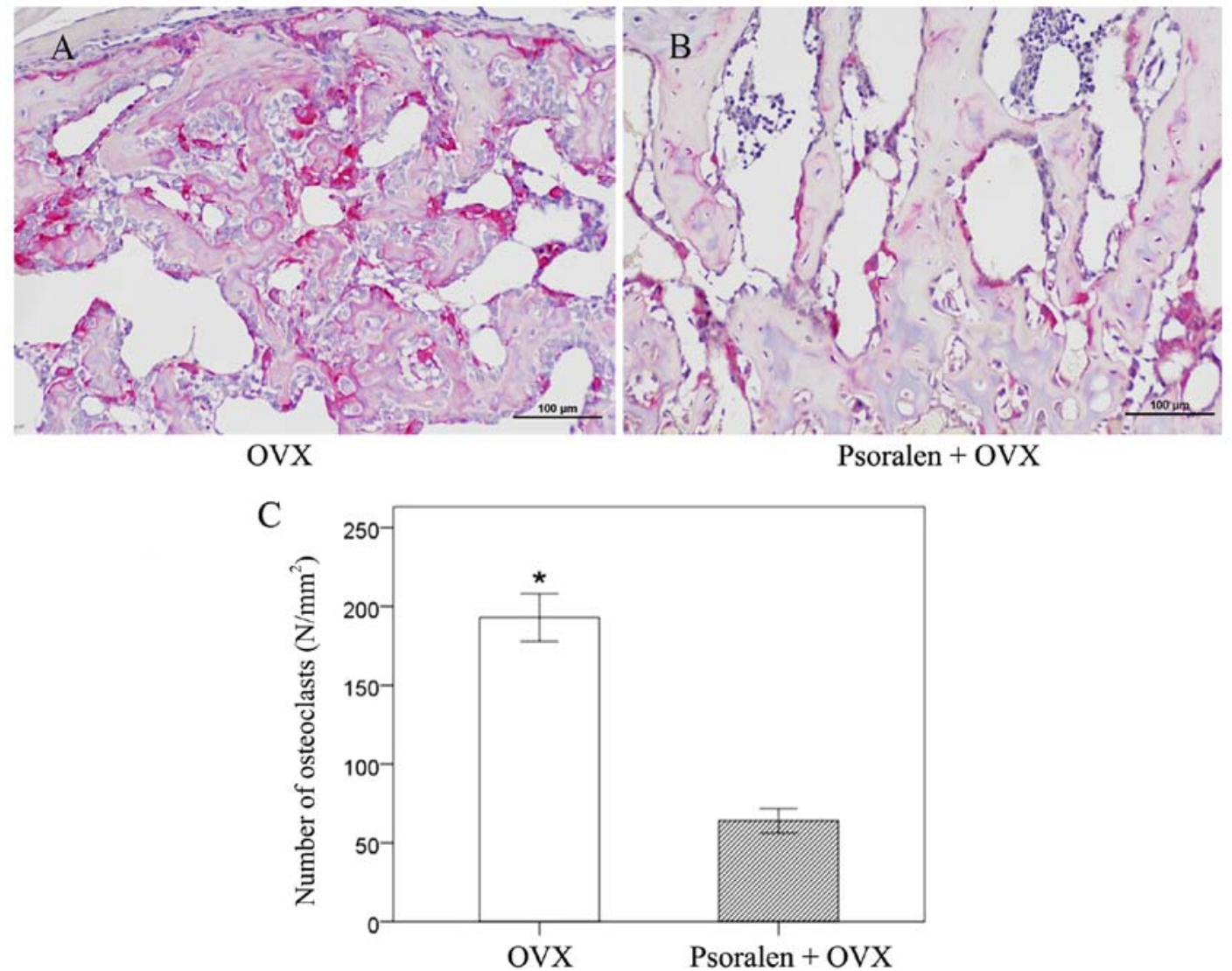

Figure 5. Osteoclast TRAP staining. Histological TRAP staining 21 days post-fracture in (A) OVX group and (B) psoralen+OVX group. Magnification, x20. (C) Mean number of TRAP-positive multinucleated giant cells in fracture callus $/ \mathrm{mm}^{2}$. Data are presented as the mean $\pm \mathrm{SD}$. "P $<0.05$ vs. psoralen+OVX group. OVX, ovariectomy; TRAP, tartrate-resistant acid phosphatase. 

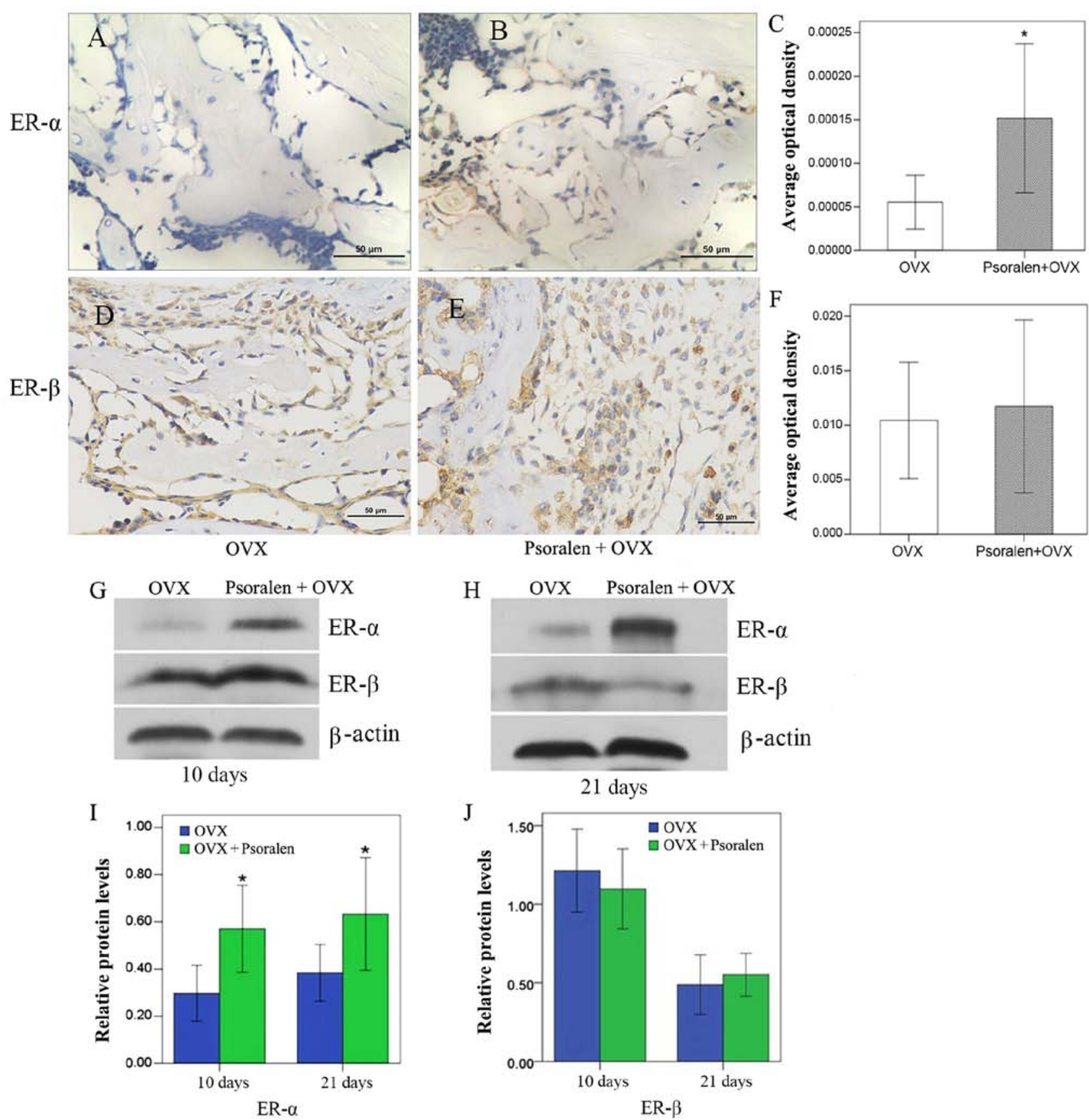

Figure 6. Expression of estrogen receptor. (A-F) Immunohistochemistry showing ER- $\alpha$ and ER- $\beta$ staining in the fracture callus of OVX and psoralen+OVX groups at 21 days post fracture. Magnification, $\mathrm{x} 40$. (G-J) Western blot analyses were performed to determine the expression levels of ER- $\alpha$ and ER- $\beta$ in OVX and psoralen+OVX groups. Data are presented as the mean $\pm \mathrm{SD}$. ${ }^{*} \mathrm{P}<0.05$ vs. OVX group. OVX, ovariectomy; ER, estrogen receptor.

increased in the psoralen+OVX group compared with the OVX group, while no difference was observed for ER- $\beta$ (Fig. 6A-F).

\section{Discussion}

Over previous years, several natural herbal drugs and plant-based bioactive molecules with anabolic potential have been examined as an alternative to traditional anti-osteoporotic medications (22). In the present study, an OVX-induced osteoporotic mouse model was established to investigate the effect of psoralen on osteoporotic fracture healing. Briefly, $\mathrm{X}$-ray and 3D micro-CT imaging revealed progressed callus formation in the psoralen+OVX group compared with the
OVX group. Furthermore, quantitative morphometry of callus structure analysis revealed that psoralen significantly increased BV and BV/TV in osteoporotic fractures in OVX mice. Moreover, a faster endochondral ossification process was observed in mice treated with psoralen compared with the control group. These findings suggest that psoralen treatment can improve fracture healing in OVX mice. In a previous study, Wong and Rabie (23) showed that psoralen treatment could increase the number of bone-forming osteoblasts and new bone formation following a bone defect in rats. Similarly, Yang et al (15) revealed that psoralen may significantly improve bone mass by stimulating osteoblastic differentiation in OVX animal models. 
At the molecular level, BMP signaling is involved in osteoporotic delayed fracture healing $(23,24)$. Our previous study indicated that the combination of locally delivered BMP-2 and systemically administered psoralen may improve bone healing in ovariectomized mice, compared with BMP-2 alone (25). Furthermore, the current study determined that psoralen may significantly increase BMP-2 expression in callus tissue. Psoralen upregulated the expression of BMP-2 and BMP-4 genes in primary osteoblasts (14). Taken together, the above results suggest that psoralen accelerates fracture healing in OVX mice, presumably by increasing bone formation and stimulating BMP-2 expression in osteoblasts.

Estrogen treatment has a direct effect on osteocytes, osteoclasts and osteoblasts, which in turn leads to the inhibition of bone resorption and maintenance of bone formation $(26,27)$. A previous study of OVX-induced osteoporotic fracture reported a moderate correlation between ER- $\alpha$ and BMP-2, thus suggesting that a high ER- $\alpha / E R-\beta$ ratio favors callus formation (28). It has been reported that phytoestrogens possess estrogenic activity and may act as a potential alternative for estrogen deficiency to prevent and treat postmenopausal osteoporosis $(29,30)$. Psoralen is a type of phytoestrogen that binds to both ER- $\alpha$ and ER- $\beta$ (31), in turn stimulating the proliferation of human breast cancer T47D cells (32). Yang et al (15) demonstrated that psoralen may reduce the gene expression and serum levels of the cytokines, tumor necrosis factor- $\alpha$ and interleukin-17, in ovariectomized rats by increasing the levels of estradiol and ER- $\beta$. In the present study, high expression of ER- $\alpha$ was observed during fracture healing in the psoralen+OVX group compared with the OVX group. This was consistent with that of a previous study (17), which indicated that psoralen as an activator, could promote the expression of ER- $\alpha$.

The ratio of $\mathrm{RANKL/OPG}$ has a role in regulating the differentiation and activation of osteoclasts, and is associated with bone loss (33). The present results showed that psoralen increased OPG mRNA expression and decreased RANKL expression to inhibit osteoclastogenesis. The histological assessment of local osteoclast activity within callus tissues showed smaller numbers of TRACP-positive multinucleated cells in the calluses of psoralen-treated mice compared with the OVX group. Gerstenfeld et al (34) showed that denosumab $(10 \mathrm{mg} / \mathrm{kg})$, which is a fully human monoclonal antibody (IgG2) that inhibits bone resorption by binding to RANKL, can almost completely eliminate TRACP-positive cells from callus tissues within 21 days, thus leading to a considerable delay in the resorption of the cartilage in a mouse fracture model.

In conclusion, these data indicated that psoralen could promote callus formation and inhibit osteoclast genesis by increasing BMP-2 and ER- $\alpha$, and increasing the OPG/RANKL ratio, respectively. Thus, it may be a useful treatment approach for osteoporotic fracture-healing complications.

\section{Acknowledgments}

Not applicable.

\section{Funding}

This work was financially supported by the Hubei Province Health and Family Planning Scientific Research Project (grant no. WJ2019M086) and the Science and Technology Development Plan Project of Jingzhou City (grant no. 2017041).

\section{Availability of data and materials}

The datasets used and/or analyzed during the current study are available from the corresponding author on reasonable request.

\section{Authors' contributions}

KBL designed the experiment, analyzed the results and revised the article. $\mathrm{KH}$ completed the experiment, statistical analysis and the writing of the first draft of the article. YQS assisted in the completion and design of the experiment, and assisted in the writing and modification of the article. XFC completed the sample detection and data analysis. FT helped produce the tissue sections and analyzed immunohistochemical staining data. FC assisted in the detection of gene expression and data analysis. QLG assisted in raising and collecting animals and analysis and interpretation of data. All authors read and approved the final manuscript.

\section{Ethics approval and consent to participate}

All animal studies, including the euthanasia procedure, were approved and performed in compliance with the regulations and guidelines of the Medical Ethical Committee of the First Affiliated Hospital of Yangtze University (approval no. 2017-060; Jingzhou, China) and conducted according to The Association for Assessment and Accreditation of Laboratory Animal Care International and the Institutional Animal Care and Use Committee guidelines.

\section{Patient consent for publication}

Not applicable.

\section{Competing interests}

The authors declare that they have no competing interests.

\section{References}

1. Compston JE, McClung MR and Leslie WD: Osteoporosis. Lancet 393: 364-376, 2019.

2. Cheung WH, Miclau T, Chow SK, Yang FF and Alt V: Fracture healing in osteoporotic bone. Injury 47 (Suppl 2): S21-S26, 2016.

3. Khosla S and Hofbauer LC: Osteoporosis treatment: Recent developments and ongoing challenges. Lancet Diabetes Endocrinol 5: 898-907, 2017.

4. Larsson S and Fazzalari NL: Anti-osteoporosis therapy and fracture healing. Arch Orthop Trauma Surg 134: 291-297, 2014.

5. Hegde V, Jo JE, Andreopoulou P and Lane JM: Effect of osteoporosis medications on fracture healing. Osteoporosis Int 27: 861-871, 2016.

6. Ha KY, Park KS, Kim SI and Kim YH: Does bisphosphonate-based anti-osteoporosis medication affect osteoporotic spinal fracture healing? Osteoporosis Int 27: 483-488, 2016. 
7. Silverman SL, Kupperman ES and Bukata SV; Members of IOFFWG: Fracture healing: A consensus report from the International Osteoporosis Foundation Fracture Working Group. Osteoporosis Int 27: 2197-2206, 2016.

8. Cosman F, de Beur SJ, LeBoff MS, Lewiecki EM, Tanner B, Randall S and Lindsay R; National Osteoporosis Foundation: Clinician's guide to prevention and treatment of osteoporosis. Osteoporosis Int 25: 2359-2381, 2014.

9. Compston J, Cooper A, Cooper C, Francis R, Kanis JA, Marsh D McCloskey EV, Reid DM, Selby P and Wilkins M; National Osteoporosis Guideline Group (NOGG): Guidelines for the diagnosis and management of osteoporosis in postmenopausal women and men from the age of 50 years in the UK. Maturitas 62 $105-108,2009$.

10. Lin J, Zhu J, Wang Y, Zhang N, Gober HJ, Qiu X, Li D and Wang L: Chinese single herbs and active ingredients for postmenopausal osteoporosis: From preclinical evidence to action mechanism. Biosci Trends 11: 496-506, 2017.

11. Leung PC and Siu WS: Herbal treatment for osteoporosis: A current review. J Tradit Complement Med 3: 82-87, 2013.

12. Salari Sharif P, Nikfar S and Abdollahi M: Prevention of bone resorption by intake of phytoestrogens in postmenopausal women: A meta-analysis. Age (Dordr) 33: 421-431, 2011.

13. Lim SH, Ha TY, Ahn J and Kim S: Estrogenic activities of Psoralea corylifolia L. seed extracts and main constituents. Phytomedicine 18: 425-430, 2011

14. Tang DZ, Yang F, Yang Z, Huang J, Shi Q, Chen D and Wang YJ: Psoralen stimulates osteoblast differentiation through activation of BMP signaling. Biochem Biophys Res Commun 405: 256-261, 2011.

15. Yang Z,Huang JH,Liu SF,Zhao YJ, ShenZY, Wang YJ and Bian Q: The osteoprotective effect of psoralen in ovariectomy-induced osteoporotic rats via stimulating the osteoblastic differentiation from bone mesenchymal stem cells. Menopause 19: 1156-1164, 2012.

16. Yuan X, Bi Y, Yan Z, Pu W, Li Y and Zhou K: Psoralen and isopsoralen ameliorate sex hormone deficiency-induced osteoporosis in female and male mice. BioMed Res Int 2016: 6869452, 2016.

17. Xin D, Wang H, Yang J, Su YF, Fan GW, Wang YF, Zhu Y and Gao XM: Phytoestrogens from Psoralea corylifolia reveal estrogen receptor-subtype selectivity. Phytomedicine 17: 126-131, 2010

18. Thulin JD, Bradfield JF, Bergdall VK, Conour LA, Grady AW, Hickman DL, Norton JN and Wallace JM: The cost of self-imposed regulatory burden in animal research. FASEB J 28 3297-3300, 2014

19. Bouxsein ML, Boyd SK, Christiansen BA, Guldberg RE, Jepsen KJ and Muller R: Guidelines for assessment of bone microstructure in rodents using micro-computed tomography. J Bone Miner Res 25: 1468-1486, 2010.

20. Naik AA, Xie C, Zuscik MJ, Kingsley P, Schwarz EM, Awad H, Guldberg R, Drissi H, Puzas JD, Boyce B et al: Reduced COX-2 expression in aged mice is associated with impaired fracture healing. J Bone Miner Res 24: 251-264, 2009.
21. Ng JJ, Wei Y, Zhou B, Bernhard J, Robinson S, Burapachaisri A, Guo XE and Vunjak-Novakovic G: Recapitulation of physiological spatiotemporal signals promotes in vitro formation of phenotypically stable human articular cartilage. Proc Nat Acad Sci USA 114: 2556-2561, 2017.

22. Jia M, Nie Y, Cao DP, Xue YY, Wang JS, Zhao L, Rahman K, Zhang QY and Qin LP: Potential antiosteoporotic agents from plants: A comprehensive review. Evid Based Complement Alternat Med 2012: 364604, 2012.

23. Wong RW and Rabie AB: Effect of psoralen on bone formation J Orthop Res 29: 158-164, 2011.

24. Chen L, Jiang W, Huang J, He BC, Zuo GW, Zhang W, Luo Q, Shi Q, Zhang BQ, Wagner ER et al: Insulin-like growth factor 2 (IGF-2) potentiates BMP-9-induced osteogenic differentiation and bone formation. J Bone Miner Res 25: 2447-2459, 2010.

25. Huang K, Wu G, Zou J and Peng S: Combination therapy with BMP-2 and psoralen enhances fracture healing in ovariectomized mice. Exp Ther Med 16: 1655-1662, 2018.

26. Khosla S, Oursler MJ and Monroe DG: Estrogen and the skeleton. Trends Endocrinol Metab 23: 576-581, 2012.

27. Wilkinson HN and Hardman MJ: The role of estrogen in cutaneous ageing and repair. Maturitas 103: 60-64, 2017.

28. Chow SK, Leung KS, Qin L, Wei F and Cheung WH: Callus formation is related to the expression ratios of estrogen receptors-alpha and -beta in ovariectomy-induced osteoporotic fracture healing. Arch Orthop Trauma Surg 134: 1405-1416, 2014.

29. Sirotkin AV and Harrath AH: Phytoestrogens and their effects. Eur J Pharmacol 741: 230-236, 2014.

30. Lagari VS and Levis S: Phytoestrogens in the prevention of postmenopausal bone loss. J Clin Densitom 16: 445-449, 2013.

31. Zhao PW, Niu JZ, Wang JF and Wang LQ: Research on phytoestrogenic effects and its mechanism of psoralen. Zhongguo Zhong Yao Za Zhi 33: 59-63, 2008 (In Chinese).

32. Soto AM, Murai JT, Siiteri PK and Sonnenschein C: Control of cell proliferation: Evidence for negative control on estrogen-sensitive T47D human breast cancer cells. Cancer Res 46: 2271-2275, 1986.

33. Catalano A, Loddo S, Bellone F, Pecora C, Lasco A and Morabito N: Pulsed electromagnetic fields modulate bone metabolism via RANKL/OPG and Wnt/ $\beta$-catenin pathways in women with postmenopausal osteoporosis: A pilot study. Bone 116: 42-46, 2018.

34. Gerstenfeld LC, Sacks DJ, Pelis M, Mason ZD, Graves DT, Barrero M, Ominsky MS, Kostenuik PJ, Morgan EF and Einhorn TA: Comparison of effects of the bisphosphonate alendronate versus the RANKL inhibitor denosumab on murine fracture healing. J Bone Miner Res 24: 196-208, 2009. International (CC BY-NC-ND 4.0) License. 